\title{
La producción de identidad de los nuevos desarrollos urbanos a través del place-based social big data: los crecimientos del área metropolitana de Madrid durante la burbuja inmobiliaria (1990-2012)
}

José Carpio-Pinedo. Universidad Politécnica de Madrid, Madrid, España. Jesús López-Baeza. HafenCity Universität Hamburg, Hamburgo, Alemania.

RESUMEN | Este trabajo propone un acercamiento al estudio de la producción de identidad y sentimiento de apropiación espacial en determinados entornos urbanos. Identidad y apropiación son estudiados desde el análisis de hitos y topónimos, utilizando para ello big data desde Foursquare, red social en la cual los usuarios publican sus visitas a lugares. Se analizan varios crecimientos urbanos de la reciente burbuja inmobiliaria en el Área Metropolitana de Madrid (1990-2012), sospechosos de falta de identidad por la reciente y rápida urbanización, así como por posibles mecanismos de simplificación del tejido urbano. El análisis valora diferentes tipos de crecimiento, revelando una correspondencia entre la tipología morfológica de los desarrollos y el tipo de lugares que constituyen hitos en ellas, así como con la aparición de topónimos, destacando ciertos lugares como elementos potenciales generadores de identidad social urbana.

PALABRAS CLAVE | desarrollo urbano, expansión urbana, sociología urbana.

ABSTRACT | This work contributes with an approach to analyze the production of identity and spatial appropriation in urban environments. To do so, the link between individual and places, and its role in the production of identity is acknowledged. This process is studied through the analysis of landmarks and toponyms - place names - with data extracted from the social network Foursquare, in which users register and publish their visits to places. Several urban growth areas developed during the recent housing bubble in the Metropolitan Area of Madrid (1990-2012), are used as case study. Due to the recent and rapid urbanization, and potential mechanisms of simplification of the urban fabric, these areas are believed to lack an identity. This work analyzes different types of growth, revealing a correspondence between the morphological typology of the new developments and the type of places that constitute landmarks in them, as well as the appearance of toponyms, to highlight certain places as potential generators of urban social identity.

KEYwORDs | urban development, urban sprawl, urban sociology. 


\section{Introducción y objetivos}

¿Qué elementos y de qué manera se relacionan los entornos urbanos con la identidad, o con el sentimiento de apropiación por parte de los ciudadanos? Se trata sin duda de una cuestión de compleja respuesta. Los procesos de producción de identidad en entornos urbanos son múltiples y de diversa naturaleza. Desde el punto de vista de la investigación y documentación, las bases de datos tradicionales no suelen recoger de manera extensiva cuestiones subjetivas como identidad o el sentimiento de apropiación.

La aparición de las tecnologías digitales, así como del gran volumen de datos producido por los usuarios en ellas - big data- supone una oportunidad en este tema. Redes sociales en las que los usuarios deciden publicar sus visitas a ciertos lugares mediante check-ins, como Foursquare, constituyen una evidente oportunidad, ya que los propios usuarios están conscientemente filtrando qué visitas hacer públicas a su red de contactos, vinculando así su identidad personal a ciertos lugares.

Por otra parte, la identidad de un entorno urbano se ha relacionado con lugares de especial diferencia, como podrían ser los de carácter histórico, patrimonial o grandes generadores de actividad. Además, los procesos de producción de identidad y de apropiación espacial se han vinculado a procesos de maduración en el tiempo. Todas estas dimensiones no se suelen dar en los entornos urbanos de reciente desarrollo, a menudo acusados de anodinos. Todavía es más problemática su ocurrencia en el caso de los tejidos producidos durante una burbuja inmobiliaria, donde cabe esperar aceleración, automatización y simplificación de los procesos, condiciones que repercuten en una aún mayor carencia de identidad.

El objetivo de este trabajo es analizar la posible existencia de una identidad social urbana en los desarrollos urbanos del área metropolitana de la ciudad de Madrid que tuvieron lugar entre 1990 y 2012, periodo conocido como la "burbuja inmobiliaria” en España. Esta identidad se entiende como potencialmente manifestada a través de hitos y topónimos -elementos simbólicos- y observable desde el big data, particularmente desde la red social Foursquare, a través de la interpretación de análisis estadístico cuantitativo (sitios registrados, visitas registradas en cada sitio, diversidad de usuarios) y cualitativo (categorías de actividad, relevancia socioeconómica, y establecimiento de nombres de lugar como topónimos). La correspondencia entre hitos y topónimos establecería la trascendencia de los lugares para la producción de identidad, y el estudio de sus características aportaría información sobre el tipo de usos y actividades asociados a esta trascendencia. Además, al centrarse en nuevos desarrollos, se trata de un análisis de las primeras etapas de producción de identidad, todavía íntimamente vinculadas a decisiones y elementos construidos por los agentes urbanizadores. Por este motivo, los resultados de los análisis pueden tener utilidad para la disciplina urbanística en términos de diseño y planeamiento.

Por otra parte, este trabajo profundiza en el estudio de los desarrollos urbanos del periodo de la burbuja inmobiliaria en Madrid, todavía poco estudiados por su reducido tiempo de vida. Por ello, se ha seleccionado un amplio número de desarrollos que abarcan diversos tipos de tejido - residencial en formatos colectivos, unifamiliares, mixtos, desarrollos mixtos residenciales y productivos, etcétera-. 
Finalmente, el estudio explora el potencial de las bases de datos de tipo social big data para los estudios urbanos, a priori de gran utilidad por su volumen, precisión geográfica y bajo coste; pero también por aportar información sobre dimensiones sociales hasta ahora escasamente capturadas.

\section{Estado del arte}

\section{La apropiación y la identidad de los entornos urbanos}

¿De dónde eres? La construcción de esta estructura pone de manifiesto la estrecha relación entre el espacio "dónde" y la propia definición del individuo "ser". Esta relación se aborda desde la psicología ambiental y social, donde el concepto de apropiación de un espacio ha sido propuesto con el fin de aportar una base teórica y empírica acerca de este vínculo entre las personas y los lugares. El proceso de apropiación espacial es constituido a través de la acción de los individuos, que incorporan el entorno como parte activa de sus procesos cognitivos y afectivos a través del apego (Vidal i Moranta \& Pol Urrútia, 2005).

Mediante la apropiación, un lugar se convierte en propio para un individuo o comunidad. Este proceso es entendido por Valera (1993) a partir del simbolismo, ligado a cómo los lugares se perciben desde su forma física tangible (Gibson, 1979; Lynch, 1960), y desde su significado intangible (Pol, 1997) y social (Stokols \& Shumaker, 1981).

En la perspectiva de la teoría espacial, Duarte (2017) habla de interdependencia entre espacio, lugar y territorio para estudiar fenómenos espaciales -y urbanos- de manera multiespectral desde una matriz epistemológica, donde convergen lo físico, psíquico y abstracto (Duarte, 2002). La literatura sobre la identidad, especialmente aquella externa a la disciplina urbanística, hace referencia a términos como placeidentity, en español "identidad espacial", evidenciando cierta intercambiabilidad en los conceptos de "lugar-place" y "espacio-space". En este contexto y en línea con Duarte (2017), entendemos "espacio" como el sustrato abstracto sobre el que se encuentran "lugar" y "territorio". El primero de ellos es construido como efecto de la percepción cognitiva del sujeto, y el segundo participa activamente de valores aplicables a los grupos sociales que se relacionan con/en él. De esta manera, al hablar de identidad espacial desde la psicología ambiental, hablamos de identidad ligada a un lugar, considerando su naturaleza procedural con componente temporal y su relación con valores afectivos. Al contrario que el concepto de territorio, el concepto de lugar hace referencia a la componente perceptual construida por las personas sobre el sustrato espacial.

La identidad social espacial -place-identity (Proshansky, 1976, 1978; Proshansky, Fabian, \& Kaminoff, 1983) o identidad urbana (Lalli, 1992)- habla de los lugares con los que las personas se relacionan. Más específicamente, el espacio no es únicamente un elemento de contexto en el que interacciones y procesos de generación de identidad ocurren; más bien, el espacio urbano es un agente activo y determinante en dichos procesos. Es desde esta perspectiva que Valera y Pol (1994) definen la identidad social espacial tomando como referencia la definición previa de identidad social relativa al autoconcepto del propio individuo, derivado de la 
consciencia de, i) pertenencia a un grupo social en conjunto con "el significado valorativo y emocional asociado a esta pertenencia” (Tajfel, 1981, p. 292), ampliada para incluir la, ii) pertenencia a entornos espaciales también con significados valorativo y emocional asociados. Proshansky, Fabian y Kaminoff (1983) ponen ambos elementos (i y ii) al mismo nivel en los procesos de construcción de la identidad del $y o$, matizando que el aspecto colectivo de los primeros viene complementado por el carácter individual de los segundos.

La relación entre individuos y grupos con el entorno no se reduce sólo a considerar este último como el marco físico donde se desarrolla la conducta sino que se traduce también en un verdadero "diálogo" simbólico en el cual el espacio transmite a los individuos unos determinados significados socialmente elaborados y éstos interpretan y reelaboran estos significados en un proceso de reconstrucción que enriquece ambas partes. (Valera \& Pol, 1994, p. 8)

Un elemento clave, según los procesos de apropiación y generación de la identidad social urbana, es la dimensión simbólica asociada a los espacios. Considerando la ciudad en su dimensión social intangible mediante una agregación de percepciones y experiencias individuales, este simbolismo se percibe y construye por los individuos principalmente a través de topónimos -el nombre de los lugares (Bonnes \& Secchiaroli, 1992) - e hitos, entendidos como espacios urbanos representativos y relevantes a nivel perceptual (Valera, 1993) y fácilmente identificables o reconocibles (Lynch, 1960). Es por ello que el estudio conjunto de hitos y topónimos puede aportar información sobre el papel de determinados lugares en la producción de la identidad social urbana. Los hitos y topónimos suponen elementos clave en torno a los cuales se desarrolla la ciudad como proceso con un valor simbólico imperativo, según la visión de autores como Lefebvre (1974) o Zukin (1996).

\section{La oportunidad del place-based social big data: Foursquare}

A partir de la posmodernidad, han surgido defensores de avanzar en la comprensión de los entornos urbanos más allá de su dimensión física tangible y objetiva. Por ejemplo, Rapoport (1977) postula que el entorno urbano ha de ser estudiado como producto social y en su vertiente humana. Cuatro décadas después, estas dimensiones intangibles de la ciudad pueden estudiarse a través de nuevas fuentes de datos, como el place-based social big data - grandes conjuntos de datos sociales geolocalizados-, alineado con las tácticas urbanas definidas por De Certeau (Lazzarini \& López Baeza, 2016) y que refleja la ciudad como producto de infinidad de fragmentos representativos de la percepción, interacciones y acciones de los usuarios, en procesos continuos (Manovich, 2017), a través de las huellas de la actividad digital de los usuarios.

La principal fuente de place-based social big data es el universo de las redes sociales. Estas se definen por la generación pública de contenido por parte del usuario y la interacción social online, utilizándose el propio contenido como plataforma en forma de red, en dirección a la folksonomía y colaboración en masa (Manovich, 2009). En múltiples ocasiones, las redes sociales llevan asociada la creación de una sensibilidad participativa (Silva, Vaz de Melo, Almeida, \& Loureiro, 2014) con 
cierto sentido de pertenencia a una comunidad o grupo social. Específicamente, las redes sociales basadas en geolocalización -las location-based social networks o LBSN- se apoyan de manera constante en el entorno espacial mediante la introducción de la tecnología GPs para convertir el lugar visitado en contenido público. El material que se comparte desde las LBSN constituye una huella digital que relaciona una interacción online con un lugar físico, por lo que su estudio puede suponer una potencial fuente de información socioespacial acerca de comportamientos, patrones y características del entorno urbano. No obstante, esta información queda claramente sesgada por un hecho: el usuario decide conscientemente qué compartir públicamente y qué no. Así, los lugares recogidos en las LBSN deben considerarse con un sesgo de valoración positiva por parte de los ciudadanos, relacionado íntimamente con la idea de apropiación.

Este trabajo se realiza a través de datos obtenidos de la red social Foursquare -entendida conjuntamente con su app complementaria Swarm-. Desde el punto de vista del interfaz del usuario, Foursquare es una LBSN que combina elementos propios de una red social -interacción, comentarios, contenido generado por el usuario- con otros tradicionalmente asociados con juegos -competición entre usuarios, puntos, rankings-, articulados en torno a los lugares físicos como elemento central. Según Saker y Evans (2016), estos tres factores justifican su crecimiento en cuanto a popularidad desde su lanzamiento en 2008. La aplicación está basada en una serie de lugares registrados, revisados y categorizados por los usuarios en los que cualquier visitante puede registrar una visita haciendo check-in. Es por ello que los datos obtenidos desde Foursquare presentan una componente humana significativa, al haber sido generados por usuarios, siendo entendidos como social-sourced data -datos de origen social- (Roick \& Heuser, 2013) o social big data.

A nivel técnico, la aplicabilidad de la red social Foursquare en los estudios urbanos queda potenciada por su gran precisión geográfica en coordenadas GPS, junto con una serie de metadatos asociados a cada lugar, como su categoría o estadísticas individuales acerca de visitas o valoraciones. Estos datos se acumulan desde el lanzamiento de la aplicación en 2008 y se ofrecen al público de manera abierta a través de una API (Application Programming Interface) en forma de sets de datos geolocalizados. La descarga de estos datos permite la visualización y análisis a cualquier escala, de cara a comprender las dinámicas urbanas desde una triple dimensión espacial-temporal-social (Kheiri, Karimipour, \& Forghani, 2015).

Los datos de Foursquare han sido ya empleados con diferentes objetivos dentro de la disciplina urbanística, desde el análisis de la diversidad de actividad económica en la ciudad (Agryzkov et al., 2015) hasta la preferencia por diferentes espacios (Martí, Serrano-Estrada, \& Nolasco-Cirugeda, 2017) o su popularidad (Komninos, Stefanis, Plessas, \& Besharat, 2013; Serrano-Estrada, Salvador, \& Álvarez Álvarez, 2014). Cerrone, López Baeza y Lehtovuori (2018) han utilizado Foursquare como base para una clasificación de actividades sobre la que también han desarrollado un índice de complejidad económica, entropía de actividades y tipos de actividad por tramo de calle (López Baeza, Cerrone, \& Männigo, 2017).

En cuanto a la componente sociológica, Zhang y Pelechrinis (2014) utilizan Foursquare para confirmar procesos de selección social vinculados a la homofilia 
-la identificación del individuo con personas que comparten condiciones sociales similares- entre grupos de amigos, basándose en el tipo y categoría espacial de lugares y visitas registradas. Además, desde el punto de vista psicosocial, uno de los potenciales de Foursquare es la capacidad para influenciar la toma de decisiones de sus usuarios, cuantificando una influencia determinante en cuestiones como qué hacer, a dónde ir, o cuándo ir (Gretzel \& Yoo, 2008).

El vínculo entre personas y lugares. El 'spatial self y los check-ins de Foursquare Son varios los estudios que han analizado la forma de utilización de Foursquare y su relación con el espacio físico de la ciudad (Jin, Zhang, Lu, \& Lin, 2016; Luke, 2005; Saker \& Evans, 2016). El hecho de compartir la localización es en sí mismo objeto de estudio para Barkhuus et al. (2008), que observan su contribución a la formación y consolidación de vínculos interpersonales. Compartir aspectos del estilo de vida, ciertos eventos o estados anímicos con el propósito de crear o mantener relaciones sociales es tomado por Schwartz y Halegoua (2014) como referencia para el desarrollo de lo que denominan spatial self, "una serie de lugares online y offline donde el individuo documenta, archiva y muestra sus experiencias y rutinas para representar aspectos de su propia identidad social a los demás" (p. 2).

El spatial self hace referencia a la práctica sociocultural intencionada de autopresentación que resulta en representaciones dinámicas, cuidadas e idealizadas de lo que el usuario es, basado en adónde va. (...). Cuando un usuario elige compartir su localización en relación a un lugar específico, está relacionándose a sí mismo con los valores y los grupos sociales que dicho lugar representa. De esta manera, los usuarios construyen su propia identidad vinculándose a la narrativa específica del espacio físico en cuestión. (Schwartz \& Halegoua, 2014, pp. 5, 7)

Sin duda, el proceso activo de construcción pública del spatial self se encuentra condicionado por la valoración previa de cada lugar que hacen las personas, y su grado de apropiación o estima mostrado hacia él. Ambos se relacionan con el valor simbólico que cada persona asigna al lugar, de acuerdo a sus condiciones personales y sociales y su deseo de "distinción" social a través de la frecuentación de ciertos lugares (Bourdieu, 1984). Así, el valor simbólico es construido por el conjunto de la sociedad, resultando en la reputación o prestigio asociado al lugar. El sesgo evidente de los datos de Foursquare es precisamente una oportunidad para caracterizar este valor simbólico de los lugares, su nivel de apropiación colectiva y su posible carácter de hito y contribución a la generación de identidad social urbana.

\section{Nuevos desarrollos urbanos y la burbuja inmobiliaria en Madrid}

¿Qué elementos y de qué manera producen la identidad de los entornos urbanos? Se trata sin duda de una cuestión de gran complejidad y especialmente difícil de abordar en el caso de los tejidos más consolidados, como los cascos históricos. Si, como afirmó Santos (1978), la ciudad es una acumulación desigual de tiempos, podemos intuir que los espacios de reciente urbanización presentan normalmente un menor grado de complejidad, pudiendo simplificar el abordaje a algunas de las cuestiones urbanas. 
La burbuja inmobiliaria española ha sido un fenómeno sin precedentes en la historia de la urbanización del país. En apenas algo más de dos décadas -19902012, aproximadamente- se han desarrollado grandes crecimientos urbanos a lo largo y ancho del territorio, sin posibilidad de maduración prolongada en el tiempo y con una probable tendencia a la estandarización o simplificación de los patrones y tipologías de urbanización. Todo ello hace que la producción de una identidad social urbana, así como el grado de apropiación, se vean cuestionados en suelos urbanos que son no solo recientes, sino el producto de procesos acelerados y simplificados que han dado como resultado paisajes urbanos homogéneos, en ocasiones calificados como "ciudad genérica" o "ciudad-basura" y "sin historia, sin referentes, sin símbolos, sin nodos de urbanidad reconocibles" (López de Lucio, 2006, p. 87) y vinculados a la idea de "no-lugares" sin identidad propia de Augé (1994).

El Área Metropolitana de Madrid (АмM) es un caso de estudio relevante dentro del conjunto español, por varios motivos. En primer lugar, la importancia de su crecimiento durante el periodo de la burbuja inmobiliaria (1990-2012). A nivel provincial, Madrid contó con la mayor urbanización de suelo en términos absolutos -casi 80.000 hectáreas-, siendo también muy importante el peso relativo -19,7\%, casi un quinto de todo el crecimiento español-. Por otra parte, los territorios urbanos de la burbuja inmobiliaria en Madrid suponen un tercio de la mancha urbana total.

Es significativo también que el crecimiento ha sido en el AMM muy variado, tanto en usos del suelo como en morfologías. Por ello, el Amm se puede tomar como un catálogo de paisajes de la burbuja inmobiliaria para toda Espańa, con la clara excepción de los crecimientos turísticos costeros.

\section{Metodología}

Caso de estudio: delimitación del crecimiento durante la burbuja inmobiliaria y selección de piezas

Para llegar a una selección de unidades de crecimiento o "piezas" urbanas, ha sido preciso realizar una serie de tareas previas. En primer lugar, se ha delimitado el crecimiento del suelo urbano a través de la base de Corine Land Cover y sus ediciones de 1990 y 2012, las cuales han servido de referencia para acotar el periodo temporal. El resultado es una "mancha" urbana continua que se ha traducido a planes urbanísticos, en su mayoría planes parciales (PP), planes de actuación urbanística (PAU) o planes especiales (PE), según los instrumentos de planeamiento español y de la Comunidad de Madrid. Esta tarea se ha realizado a través del Sistema de Información Geográfica (sIG) de Planeamiento de la Comunidad de Madrid como base, completado y corregido con una consulta pormenorizada de la documentación y cartografía de los planes urbanísticos. En paralelo, se ha aumentado el grado de detalle geográfico de la mancha de Corine Land Cover mediante una comprobación con la foto aérea de 1991 (disponible en 'Nomecalles' - Nomenclátor y Callejero de la Comunidad de Madrid) como muestra la figura 1.

De entre todas las piezas resultantes, se han seleccionado 43 con la intención de describir toda la variedad morfológica y funcional del conjunto, intentando también representar crecimientos en todas las posiciones geográficas del AMM, para 
así evitar sesgos derivados de la gran segregación socioeconómica dada (Leal \& Sorando, 2015).

Para un análisis por tipología de las piezas, se ha tomado la clasificación del proyecto I+D+i LUrB "Lecciones Urbanísticas de la Burbuja Inmobiliaria" (Pozueta Echavarri, Benito, Carpio, González, \& Gómez, en prensa; Pozueta, Carpio, Tumini, \& González, en prensa) recogida en la tabla 1.

\begin{tabular}{|l|l|}
\hline \multicolumn{1}{|c|}{ CóDIGo } & \multicolumn{1}{c|}{ DESCRIPCIÓN } \\
\hline MX & Mixto residencial y no residencial \\
\hline RC-MC & Residencial: vivienda colectiva en manzana cerrada \\
\hline RC-B & Residencial: vivienda colectiva en bloques \\
\hline RM & Residencial: mixto plurifamiliar y unifamiliar \\
\hline RU & Residencial: unifamiliar \\
\hline
\end{tabular}

TABLA I | Clasificación de los tejidos estudiados según su tipología de planeamiento

FUENTE: ELABORACIÓN PROPIA A PARTIR DEL PROYECTO "LECCIONES URBANÍSTICAS DE LA BURBUjA INMOBILIARIA", DE POZUETA ECHAVARRI ET AL. (EN PRENSA)

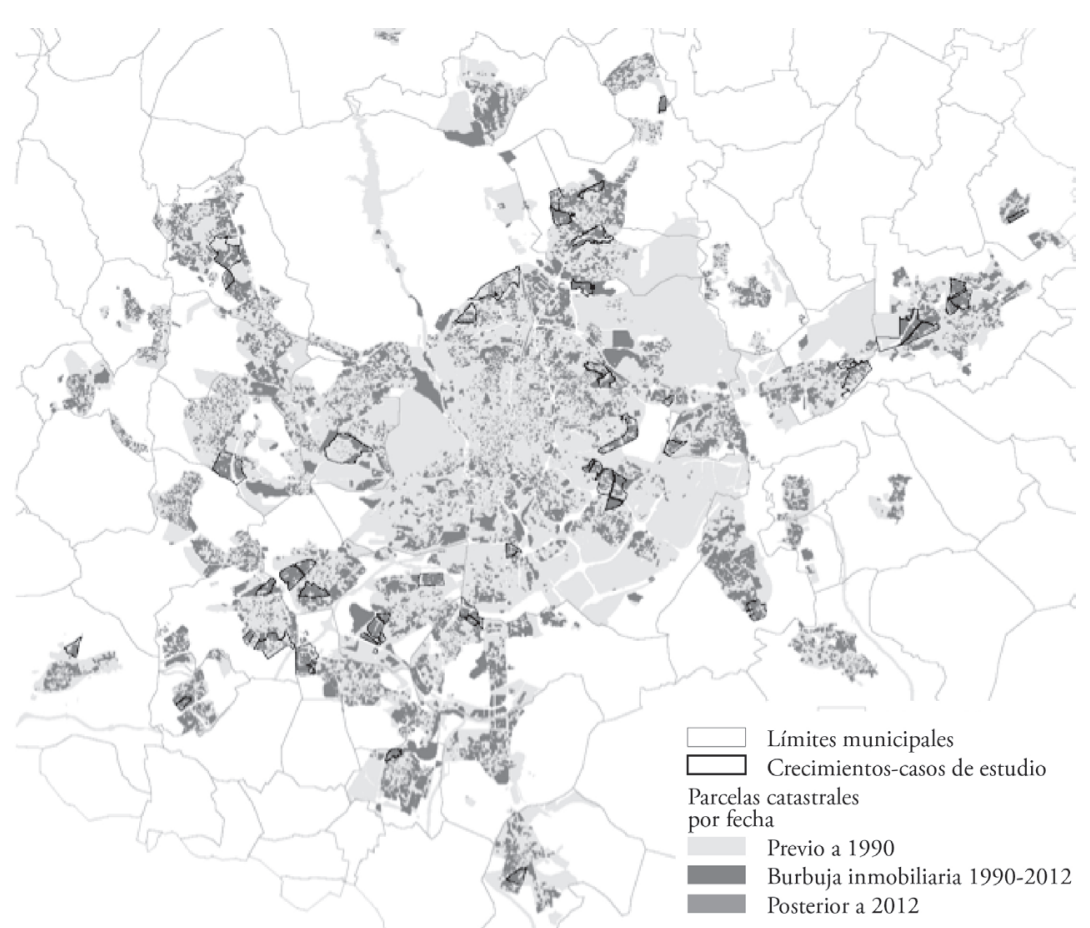

FIGURA I | Localización e identificación de las piezas seleccionadas como casos de estudio

FUENTE: ELABORACIÓN PROPIA 


\section{La base de datos de Foursquare. Características, descarga y columnas}

Los sets de datos descargados desde la API pública de Foursquare "Places API" representan lugares físicos registrados en un entorno urbano determinado, con metadatos asociados. La descarga de datos se realiza mediante consultas cíclicas y repetidas a la API, estableciendo como parámetro el contexto espacial mediante límites en forma de coordenadas GPS, como longitudes y latitudes extremas. Los metadatos asociados a cada lugar son variados. Para el presente trabajo se toman las coordenadas espaciales, el nombre del lugar, las categorías de actividad y las estadísticas relativas a número de usuarios que visitan el lugar (users count) y número total de publicaciones de visitas en cada lugar (check-ins). No todas las personas que visitan un lugar deciden publicar su visita en su red social, siendo este hecho interesante para valorar la popularidad de un lugar. Otros datos disponibles son el nombre, la dirección, las subcategorías de actividad, las fotografías y la valoración media dada por los usuarios.

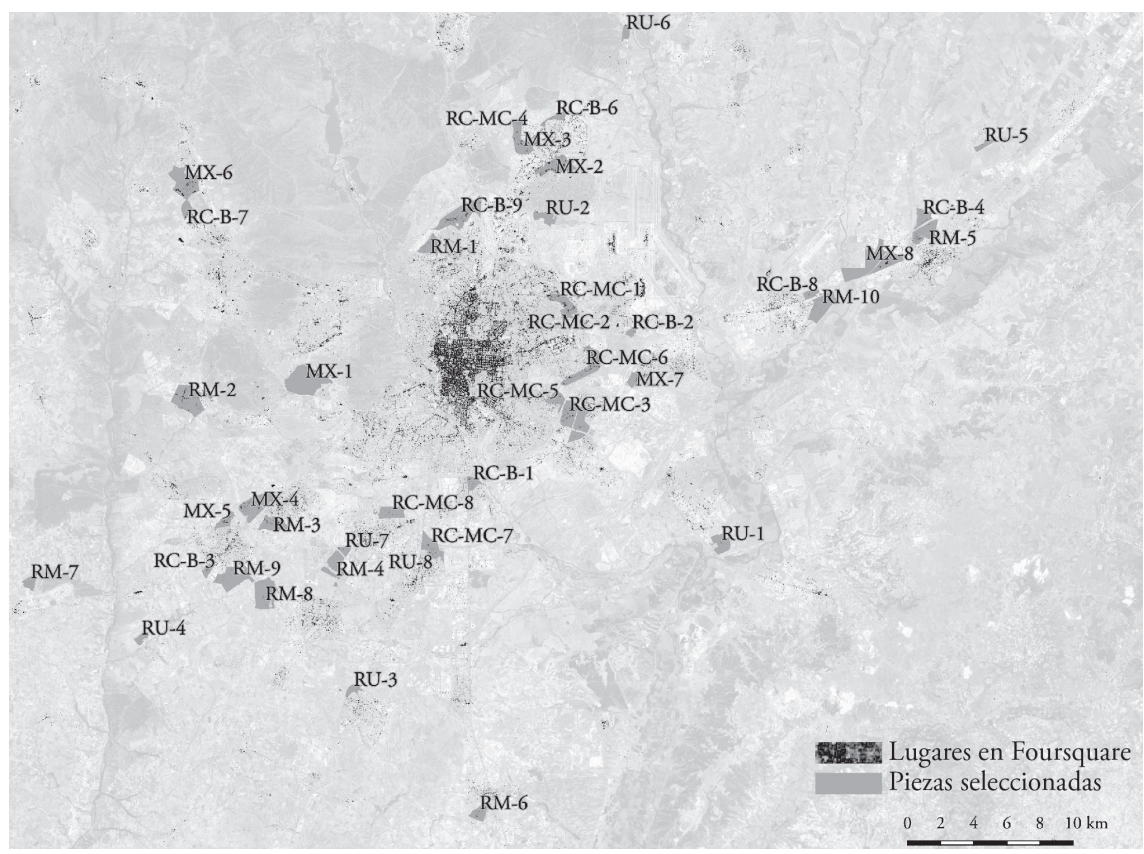

FIgURA 2 | Área de descarga del set de datos principal. Representados los lugares registrados en Foursquare contenidos en el set de datos

FUENTE: ELABORACIÓN PROPIA

La base de datos de Foursquare descargada para este trabajo ha abarcado la Comunidad de Madrid y la compone un total de 94.010 lugares, donde se han registrado 16.553 .533 check-ins. La descarga de datos se realizó en octubre de 2018, siendo los datos cuantitativos -número de check-ins, número de usuariosde carácter acumulativo desde el registro de cada lugar en la red social, fecha potencialmente coincidente con, i) la implementación de Foursquare en 2009 o, 
ii) la fecha aproximada de apertura al público del lugar en cuestión. Las 43 piezas urbanas seleccionadas como casos de estudio incluyen en su contorno 4264 lugares registrados y, como se puede observar en la figura 2, no se sitúan en los entornos con mayor concentración de lugares en la base de Foursquare. Esta observación responde tanto a su carácter periférico o suburbano, como a su reciente desarrollo. Estas 43 piezas recogen apenas el 4,5\% de lugares y de check-ins del conjunto de la Comunidad de Madrid.

\section{Índices}

\section{Tipos de actividad}

El estudio de las tipologías de actividades dentro de la disciplina urbanística ha sido abordado previamente centrándose en la función primaria de los lugares, la correspondencia con las categorías normativas de usos del suelo, la importancia de la actividad en la rutina diaria o su grado de necesidad (Ben-akiva \& Bowman, 1998; Buttimer \& Seamon, 1980; Gehl, 1987; Jiang, Ferreira, \& González, 2012; Seamon, 1979). Estas referencias son tomadas por Cerrone et al. (2018) en el desarrollo de una clasificación de actividades en el espacio urbano basada de manera primaria en la necesidad de acción -con origen en la primera clasificación de Gehl (1987) en actividades necesarias y opcionales- y que intenta abarcar todo el espectro de actividades urbanas, independientemente de la naturaleza del espacio en el que se realizan.

Cerrone et al. (2018) proponen una clasificación basada en dos categorías ("Necesaria" y "Opcional") y diez subcategorías, cinco en cada uno de esos dos grupos, recogidas en la tabla 2. A estas diez subcategorías se ańade otra sin definir ("Otro"), la cual engloba aquellos lugares sin una actividad primaria definida, como barrios, calles o edificios entendidos como "un lugar" en su conjunto. Estos lugares existen en la base de datos, también registran visitas por los usuarios y son accesibles a la búsqueda por nombre, por lo que a pesar de no describir una actividad concreta, su utilidad en el estudio de hitos y toponimia no queda en absoluto descartada.

\begin{tabular}{|l|l|}
\hline \multicolumn{1}{|c|}{ NECESARIA } & \multicolumn{1}{c|}{ OPCIONAL } \\
\hline Salud & Ocio \\
\hline Alimentación & Social \\
\hline Trabajo & Cívico \\
\hline Movilidad & Consumo \\
\hline Educación & Cuidado Personal \\
\hline
\end{tabular}

TABla 2 | Tipos de actividad definidos por Cerrone et al. (2018)

FUENTE: ELABORACIÓN PROPIA

El caso particular de Foursquare contribuye a la definición de la actividad reali$z a d a$, entendida como el resultado de la intersección entre oferta y demanda. En otras palabras, dado un conjunto de actividades que el entorno urbano permite 
realizar (oferta o potencial) y otro conjunto de actividades que se quieren realizar (demanda), se trata de las actividades que finalmente se realizan. La clasificación de las actividades en Foursquare, junto con el estudio cuantitativo de, i) los lugares registrados y, ii) sus estadísticas -número de usuarios y número de check-ins obtenidos-, permiten avanzar en el estudio de la génesis de hitos y topónimos.

\section{Hitos}

Para operacionalizar metodológicamente los hitos, entendidos como espacios relevantes, reconocibles y reconocidos, se identifican aquellos lugares de Foursquare sobre los que se ha publicado un mayor número de visitas (check-ins). Un espacio relevante en la red social Foursquare sería aquel que reúne una cantidad significativa y sobresaliente de visitas compartidas a las redes sociales, con respecto a los valores medios del entorno.

\section{Topónimos}

Los posibles topónimos se han obtenido mediante un conteo de frecuencia de repetición de palabras sobre el set de datos de Foursquare, en el campo referido al nombre de los lugares. En otras palabras, si en un mismo crecimiento aparece una estación de tren de nombre " $X$ ", una panadería " $X$ " $y$ una escuela de primaria " $X$ ", se puede valorar que hay tres lugares con el nombre " $X$ " como posible topónimo en el área de estudio. A su vez, este topónimo podría ser un elemento de constitución de identidad social urbana de dicha área.

En este sentido, la fortuna de ciertos nombres como topónimos puede ser el resultado de múltiples y distintas casuísticas. Por ejemplo, podría ser que el nombre del plan urbanístico se haya repetido y popularizado en los medios de comunicación o a través de la publicidad de numerosas promociones inmobiliarias, o que haya llegado a bautizar las estaciones de transporte público, en caso de haberlas. $\mathrm{O}$ quizás puede que los ciudadanos hayan identificado el barrio con algún espacio o equipamiento singular, por un proceso de metonimia. $\mathrm{O}$ puede ser que nada de esto haya ocurrido.

Para la identificación de topónimos, se tomaron todas las palabras encontradas en el nombre de los lugares en el interior de la delimitación establecida de las piezas, y repetidas en la base de datos tres o más veces -en total 700 palabras-. Esta lista se ha revisado palabra por palabra para filtrar aquellas que son efectivamente topónimos o nombres de un lugar, pudiendo tratarse del nombre de un barrio, de todo un municipio o de una vía principal, pero no como un local específico, como "Marisol”, o una tipología genérica, como "zapatería".

\section{Resultados y discusión}

\section{Tipo de actividades: Estadísticas generales}

De los 4264 lugares registrados en los desarrollos urbanos estudiados podemos extraer diversas características generales, partiendo de la tipología de actividad de los lugares, según el trabajo desarrollado por Cerrone et al. (2018). En cuanto a tendencias generales, la tabla 3 muestra la agregación de datos cuantitativos por cada una de las categorías, presentes en todas las piezas de estudio. 


\begin{tabular}{|l|c|c|c|c|}
\hline \multicolumn{1}{|c|}{ CATEGORÍA } & N. $^{\mathbf{0}}$ DE REGISTROS O LUGARES & \multicolumn{2}{c|}{ N. $^{\mathbf{0}}$ DE CHECK-INS } \\
\hline Consumo & 773 & $19,43 \%$ & 146.888 & $19,99 \%$ \\
\hline Social & 893 & $22,45 \%$ & 144.384 & $19,65 \%$ \\
\hline Trabajo & 344 & $8,65 \%$ & 121.804 & $16,57 \%$ \\
\hline Ocio & 399 & $10,03 \%$ & 86.136 & $11,72 \%$ \\
\hline Cuidado personal & 307 & $7,72 \%$ & 74.832 & $10,18 \%$ \\
\hline Otra & 241 & $6,06 \%$ & 48.253 & $6,57 \%$ \\
\hline Alimentación & 370 & $9,30 \%$ & 47.332 & $6,44 \%$ \\
\hline Movilidad & 114 & $2,87 \%$ & 24.714 & $3,36 \%$ \\
\hline Educación & 120 & $3,02 \%$ & 15.791 & $2,15 \%$ \\
\hline Salud & 162 & $4,07 \%$ & 13.092 & $1,78 \%$ \\
\hline Cívico & 255 & $6,41 \%$ & 11.650 & $1,59 \%$ \\
\hline
\end{tabular}

TABLA 3 | Cantidad de lugares registrados y cantidad de check-ins acumulados por cada categoría según la clasificación de lugares de Cerrone et al. (2018)

FUENTE: ELABORACIÓN PROPIA

En cuanto a la cantidad de lugares y check-ins por cada tipología de tejido, la figura 3 muestra una tendencia de las piezas de tipo mixto residencial-no residencial (Mx) a contener un mayor nivel de actividad, debido a la mayor presencia de actividad comercial y empresarial. También las piezas de residencial colectivo en manzanas cerradas llegan a números de lugares y de check-ins significativos, quedando por detrás las piezas residenciales mixtas unifamiliar-colectivo (con la excepción del Ensanche Oeste de Boadilla), y muy por detrás las piezas de vivienda colectiva en bloques y unifamiliares (RCB y RU). Desde el punto de vista morfológico, la menor densidad de lugares y visitas registradas en piezas de unifamiliares se corresponde con la igualmente baja densidad residencial y edificada. No obstante, las diferencias entre tipologías residenciales colectivas son interesantes y se corresponden con la literatura que apunta hacia una mejor y más detallada definición del "paisaje urbano" en tejidos de manzana cerrada -que se realiza a través de la recuperación de la calle corredor- en contraste con el espacio público abundante e indefinido característico de los tejidos de bloques (López de Lucio, 2013; López de Lucio \& Hernández Aja, 1995; Panerai, Castex \& Depaule, 1997).

De manera general, aquellas piezas con un mayor número de lugares registrados tienden a tener un mayor número de check-ins acumulados, con dos excepciones. En primer lugar, el Ensanche Este de San Blas-Las Rosas (RCMC6) presenta un escaso número de check-ins en comparación con el gran número de lugares registrados. El ejemplo opuesto es Somosaguas Sur (MXI), con escaso número de lugares pero gran cantidad de check-ins, apuntando al alto valor simbólico o prestigio que ellos tendrían. Se trata de una zona distinguida, que incluye la urbanización La Finca, reconocida por su lujo y domicilios de celebridades. Por eso, no sorprende que todo usuario de Foursquare que pase por allí decida compartir su visita a su red social. 


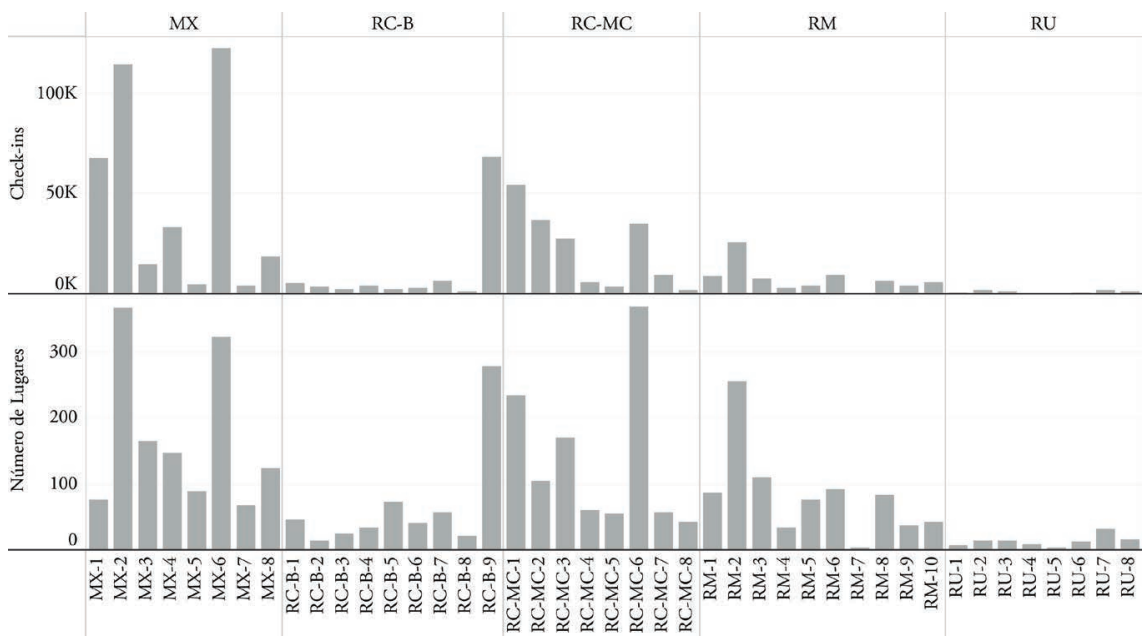

FIGURA 3 | Número de check-ins y lugares registrados en cada pieza, estando las piezas agrupadas por tipología

FUENTE: ELABORACIÓN PROPIA

\section{Hitos}

La relación entre cantidad total de veces que un lugar ha sido visitado (check-ins) y cantidad de usuarios diferentes que visitan los lugares (users count) ha permitido identificar lugares de rutina y lugares de ocio. En los gráficos que muestra la figura 4, el eje vertical representa la cantidad de usuarios que visitan cada lugar individual, y el eje horizontal da cuenta del número de veces que el lugar ha sido visitado en cómputo general. En este contexto, tomando como referencia aquellos lugares individuales con un número de check-ins superior a 500 -queda excluida la categoría "Cívico"-, observamos que las líneas con mayor pendiente corresponden a categorías relacionadas con actividades opcionales recreacionales. Aquellas con menor pendiente representan actividades habitualmente rutinarias (trabajo, estudio).

A partir de la distribución de los puntos en los gráficos de la figura 4, y tomando en cuenta ambos valores -número de check-ins y número de usuarios-, los lugares del set de datos quedan distribuidos gradualmente. En referencia a aquellos lugares con mayor número general tanto de usuarios que los han visitado como de visitas (check-ins) registradas en total, se observa que, salvo tres excepciones, ${ }^{1}$ se trata de grandes superficies comerciales, de ocio o equipamientos de relevancia a escala urbana. Por el contrario, aquellos más cercanos a los ejes representan lugares con relevancia únicamente en la escala local, como pequeńas tiendas, restaurantes, bares, etcétera.

1 Una franquicia (Starbucks en el Parque Empresarial de las Rozas/MX-6), una sede empresarial (Orange en Somosaguas Sur/MX-I) y un topónimo (Montecarmelo). 


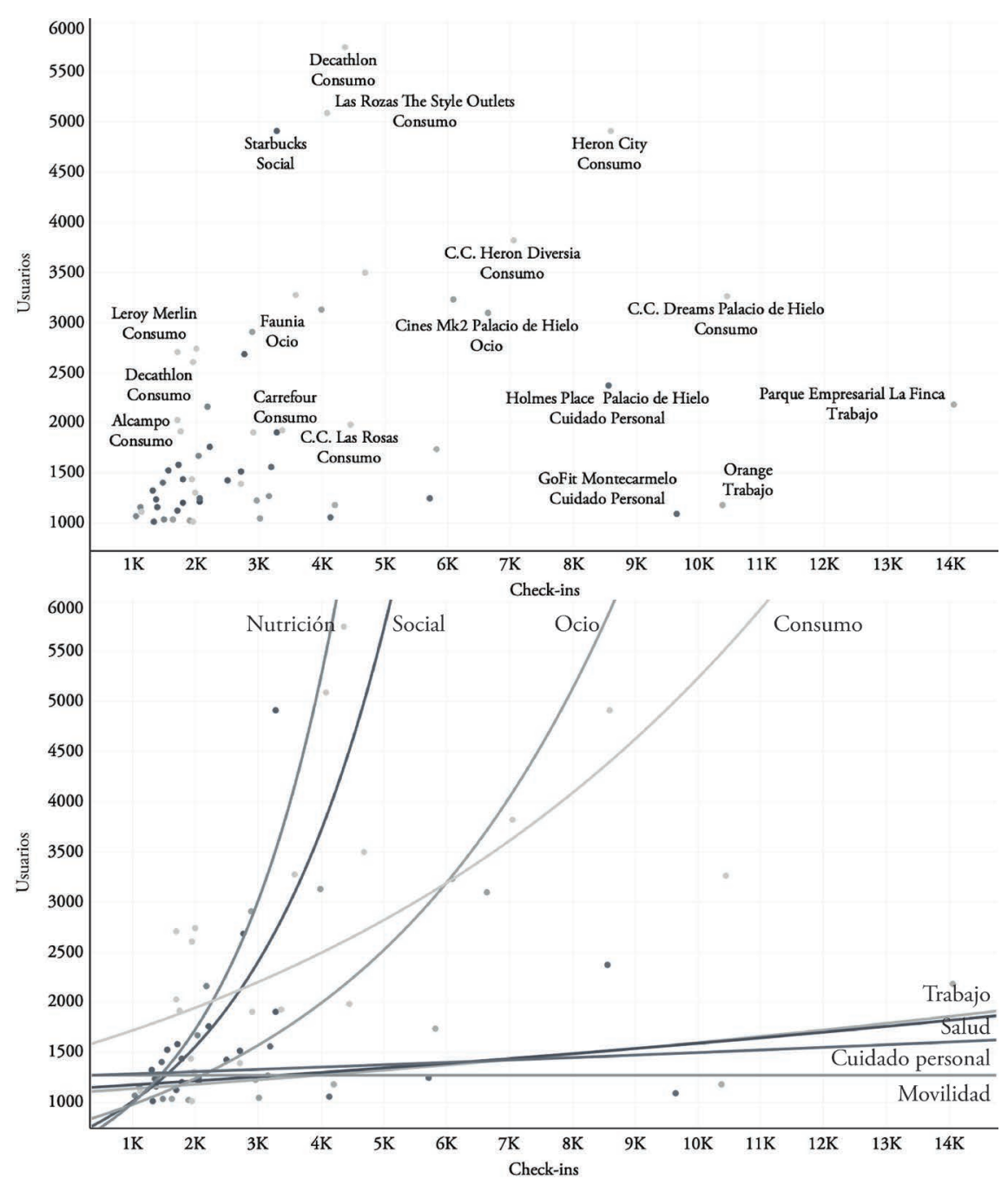

FIGURA 4 | Representaciones de lugares individuales en el set de datos con número de check-ins, en relación al número de usuarios que los visitan. (superior) Conjunto de datos según su categoría de actividad; (inferior) Zoom sobre el conjunto central de puntos. El Centro Comercial Las Rozas Village -con 21.553 check-ins y 19.833 usuarios- está excluido de ambas representaciones, pero tenido en cuenta en el estudio 


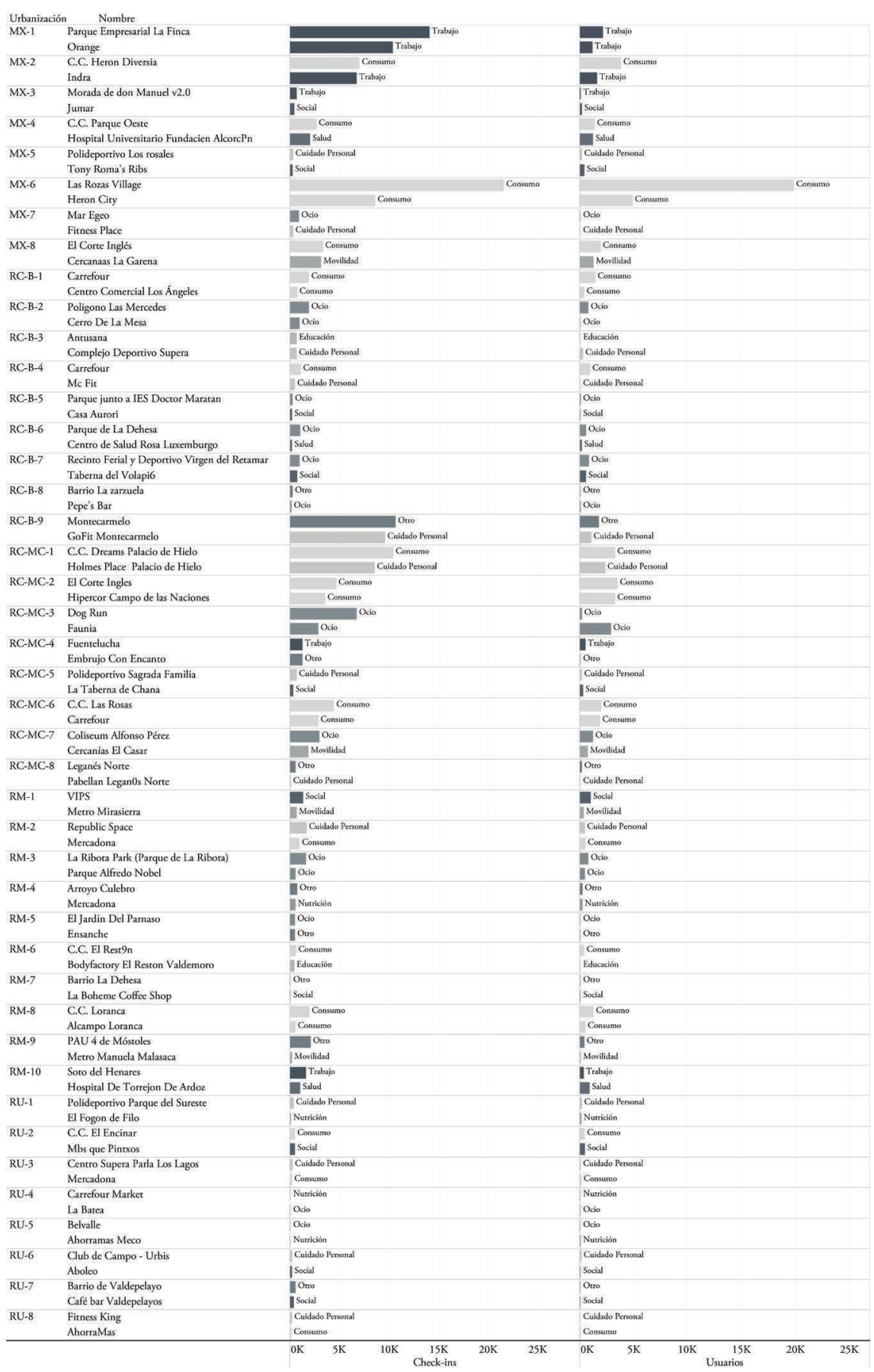

FIGURA 5 | Top 3 lugares más visitados en cada una de las piezas

FUENTE: ELABORACIÓN PROPIA 
Por otra parte, se han analizado los lugares más relevantes por cada pieza, realizándose el análisis de algunos patrones por tipología urbanística (figura 5 y tabla 4). En primer lugar, como era previsible, los tejidos de vivienda unifamiliar destacan por el escaso volumen de actividad en Foursquare. En el extremo opuesto, se confirma que son los tejidos mixtos (residenciales-otros usos) y los residenciales en formatos colectivos los que obtienen mayores valores en las variables de Foursquare. De entre los residenciales, también se corrobora que los tejidos de manzana cerrada obtienen de media un mayor número de usuarios y check-ins. No obstante, cabe subrayar el gran contraste que puede producirse entre piezas diferentes de una misma tipología. Entre las piezas mixtas, encontramos algunas como el Parque Empresarial de Las Rozas (MX6) o Somosaguas Sur (MXI), con varios de los mayores hitos de entre todos los tejidos analizados, llegando incluso a superar los 20.000 check-ins, como populares centros comerciales o la sede de importantes empresas. Sin embargo, también son mixtas algunas piezas como Casablanca-Espino del Cuquillo en Alcobendas (MX3), Los Rosales en Móstoles (MX5) o el Sector Residencial-Industrial de Coslada(MX7), donde los resultados en Foursquare son muy pobres.

Lo mismo ocurre en los tejidos de residencial colectivo, donde se dan contrastes fuertes entre piezas con hitos muy populares en Foursquare -en Madrid, Montecarmelo (RCB9) o Arroyo del Fresno (RCMCI)-, junto a otras carentes de hitos de cierta relevancia-Sector 41 de Alcalá (RCB5) o Leganés Norte (RCMC8)-.

\begin{tabular}{|c|c|c|c|c|c|c|}
\hline & \multirow{3}{*}{$\begin{array}{c}\mathbf{M X} \\
1 \\
\end{array}$} & \multirow{3}{*}{$\begin{array}{c}\text { RC-B } \\
1\end{array}$} & \multirow[b]{2}{*}{ RC-MC } & \multirow{3}{*}{$\begin{array}{c}\mathbf{R M} \\
1\end{array}$} & \multirow{3}{*}{$\begin{array}{r}\mathbf{R U} \\
1\end{array}$} \\
\hline & & & & & & \\
\hline \multirow{5}{*}{ Necesaria } & Salud & & & & & \\
\hline & Alimentación & & & & 2 & 3 \\
\hline & Trabajo & 6 & & 1 & 1 & 1 \\
\hline & Movilidad & 1 & & 1 & 3 & \\
\hline & Educación & & 1 & 1 & 1 & 1 \\
\hline \multirow{5}{*}{ Opcional } & Ocio & 2 & 7 & 4 & 8 & 2 \\
\hline & Social & 5 & 7 & 4 & 4 & 6 \\
\hline & Cívico & & & & & \\
\hline & Consumo & 7 & 4 & 6 & 5 & 3 \\
\hline & Cuidado personal & 2 & 4 & 5 & 1 & 6 \\
\hline
\end{tabular}

TABLA 4 Número de hitos por tipología urbanística y por tipo de actividad, entre el conjunto de los tres hitos más relevantes en cada pieza

FUENTE: ELABORACIÓN PROPIA

Entrando a valorar a partir de los tipos de actividad, un primer hecho necesario de comentar es el mucho mayor número de hitos asociados a actividades opcionales, respecto a las necesarias. Esto entra en la lógica de la excepcionalidad y de la construcción de la identidad personal a partir de la elección voluntaria de actividades y de lugares, en relación al spatial self presentado en la literatura revisada. 
Por tanto, los hitos son en su mayoría lugares de actividades voluntarias, asociadas al consumo y al ocio, al encuentro social o al cuidado personal. Destacan los grandes lugares de consumo, como los centros comerciales, que aparecen como hitos de gran importancia en algunas piezas mixtas y de manzana cerrada, pero pudiéndose encontrar ejemplos en todos los tipos urbanísticos. Además, es habitual que estos espacios comerciales sean el hito principal de la pieza. En relación también con la producción de la identidad a través de topónimos, cabe destacar que no solo grandes centros comerciales con nombre propio aparecen como hitos: también establecimientos de cadenas o franquicias (Decathlon, Carrefour o El Corte Inglés) se encuentran entre los principales hitos de algunas piezas. Lejos de ser ignorados por la ciudadanía como nolugares (Augé, 1994) repetidos y sin identidad, en la escala local de los nuevos barrios de la periferia están consiguiendo funcionar como hitos y aportar a los nuevos tejidos un valor simbólico consolidado previamente en otros entornos urbanos.

Entre el resto de categorías opcionales, aparecen con frecuencia los parques y zonas verdes, así como espacios de ejercicio físico y deporte, tanto públicos (centros polideportivos municipales) como privados (clubes deportivos). Si bien estos últimos sin duda parecen desempeñar un papel más importante en la estrategia de distinción social de ciertas personas (Bourdieu, 1984), la asistencia a todos ellos publicada en redes sociales refuerza la creación del personaje como alguien que cuida su salud y estética.

Los hitos asociados a actividades básicas o necesarias son mucho menos relevantes desde el punto de vista cuantitativo. Solo sobresalen los lugares de trabajo en el caso de las piezas mixtas residenciales-otros usos, que acogen las sedes de grandes empresas (Orange, Microsoft, Accenture, Indra o Hewlett-Packard). No obstante, e interesante desde el punto de vista de la identidad y los topónimos, en un par de piezas hemos encontrado que el hito lo configura la propia agrupación de empresas (Parque Empresarial La Finca y Soto del Henares).

Los equipamientos sanitarios se constituyen como hitos en cuatro de las piezas analizadas, siendo todos ellos públicos, a pesar de que algunos, como los hospitales de Torrejón y de Alcorcón, sean de gestión privada externalizada, modelo potenciado en Madrid por los gobiernos regionales de derechas. Entre los escasos equipamientos educativos que en los análisis aparecen como hitos, excepto uno, todos son centros privados, y se hace de nuevo pertinente relacionar esta situación con las estrategias de distinción social. En este sentido, cabe apuntar que la Comunidad de Madrid ha sido recientemente considerada como uno de los entornos con mayor segregación escolar en Europa (Murillo \& Martínez-Garrido, 2018) y, en un contexto de tal desigualdad, puede interesar aclarar a nuestra red social en qué extremo nos situamos.

Por otra parte, solo en cinco piezas se ha detectado un hito relevante asociado a la movilidad. En todos los casos se trata de una estación de transporte público de cierta entidad (trenes regionales o metro). Cabe destacar la estación de La Garena, en el crecimiento homónimo (MX-8), que presenta una cantidad de check-ins por encima del valor promedio. La Garena es un crecimiento urbano planificado y desarrollado con una vinculación extraordinaria a dicha estación, a la manera de 
los transit-oriented developments o TOD (Lamíquiz, Pozueta, Benito, \& González, 2017). Por lo tanto, la relevancia de la estación de cercanías en el tejido social -así como el hecho de que el nombre de la estación sea compartido con el topónimo del barrio- la constituye como hito y elemento generador de identidad social urbana.

Por último, cabe hacer un apunte sobre la posibilidad de hitos asociados a la más básica de las actividades: la alimentación. Desde la clasificación primaria de Cerrone et al. (2018), la categoría "Alimentación" se considera actividad necesaria desde la reflexión de que estos lugares -restaurantes de fast food, supermercados, hipermercados y tiendas de alimentación- no son lugares de socialización, sino que únicamente sirven al propósito de nutrición cuando esta es necesaria. Al mismo tiempo, los restaurantes especializados en otros tipos de cocina son considerados como actividad opcional en el ámbito de la socialización, ya que no sirven a la función de la nutrición necesaria, sino que atienden al propósito de interacción social. No obstante, con base en la observación de los datos de la figura 4 relativos a la pendiente de la línea de tendencia, puede entenderse que las actividades categorizadas como "Alimentación” presentan una tendencia similar a las actividades de tipo "Social" y "Ocio", a partir de lo cual podría discutirse el papel de los restaurantes de fast food (McDonald's, Burger King), sobre todo para ciertos colectivos (adolescentes, familias, etcétera) y de las grandes áreas comerciales de alimentación (Mercadona, Alcampo, Hipercor), no únicamente como lugares para la nutrición necesaria, sino también como lugares, en algunos casos, de interacción social. Pero es importante, además, resaltar cómo todas las marcas y franquicias señaladas conectan estos tejidos de nueva ciudad con el conjunto de lugares reconocibles en el resto de la ciudad y por parte del conjunto de la sociedad, aportando el valor simbólico ya consolidado al que se apuntaba anteriormente.

\section{Topónimos}

Los resultados del estudio de topónimos son heterogéneos, con varias piezas donde no se ha encontrado ningún topónimo consistente. De entre las 43 analizadas, son solo cinco las piezas que cuentan con un claro topónimo que las hace reconocibles entre la ciudadanía: Montecarmelo (RC-B-9), Loranca (RM-8), Las Rosas (RC-MC6), Valdebernardo (RC-MC-3) y La Garena (MX-8). En ellas se ha producido una identidad urbana clara y distintiva, haciendo que el topónimo aparezca hoy en una variedad de espacios. Por ejemplo, para el caso de Montecarmelo, se ha encontrado lugares como Farmacia Montecarmelo, Restaurante Montecarmelo, Clínica Montecarmelo o Floristería de Montecarmelo.

¿Qué rasgos comunes encontramos en estas cinco piezas? Todas ellas cuentan, en primer lugar, con una amplia parte de vivienda en formatos colectivos. Igualmente, es un denominador común la presencia de una estación de transporte con ese nombre, ya sea de metro en los cuatro primeros casos o de trenes metropolitanos de cercanías en el caso de La Garena. Otro rasgo morfológico común es que cuatro de estas cinco piezas presentan un cierto nivel de aislamiento respecto de los tejidos urbanos vecinos, estando significativamente acotadas por espacios libres o sin actividad o por autovías o líneas férreas que actúan como frontera. Solo el caso de Las Rosas presenta una mayor conectividad con el vecino barrio de San Blas, si bien 
el contraste morfológico, de calidad y antigüedad de las edificaciones se hace muy evidente. Por otra parte, la última expansión de la Línea 2 de metro se publicitó ampliamente en los medios de comunicación como "ampliación a Las Rosas", con titulares en prensa que valoraban la nueva cobertura para "los vecinos de Las Rosas".

Entre los casos analizados, también encontramos un caso donde no ha aparecido un topónimo que aporte identidad a la pieza, y los más utilizados han sido los nombres de las dos vías que estructuran la pieza. Se trata del desarrollo urbano de Los Llanos (RC-MC-I), donde la calle Silvano o la avenida de Machupichu son las que han dado nombre en el imaginario colectivo a la pieza.

Finalmente, entre los resultados aparecen habitualmente los topónimos correspondientes al municipio donde se localiza la nueva pieza (Getafe, Alcalá de Henares o Alcobendas, entre otros, recogidos en la figura 6). Esto podría apuntar a cómo, por medio del nombre de los equipamientos, comercios y empresas, se está insistiendo en el vínculo y la pertenencia del nuevo desarrollo a la ciudad existente. Entre todos los casos, destaca el éxito del topónimo Boadilla en la pieza del Ensanche de Boadilla (RM-2), siendo un desarrollo que supera ampliamente en extensión al casco urbano preexistente.

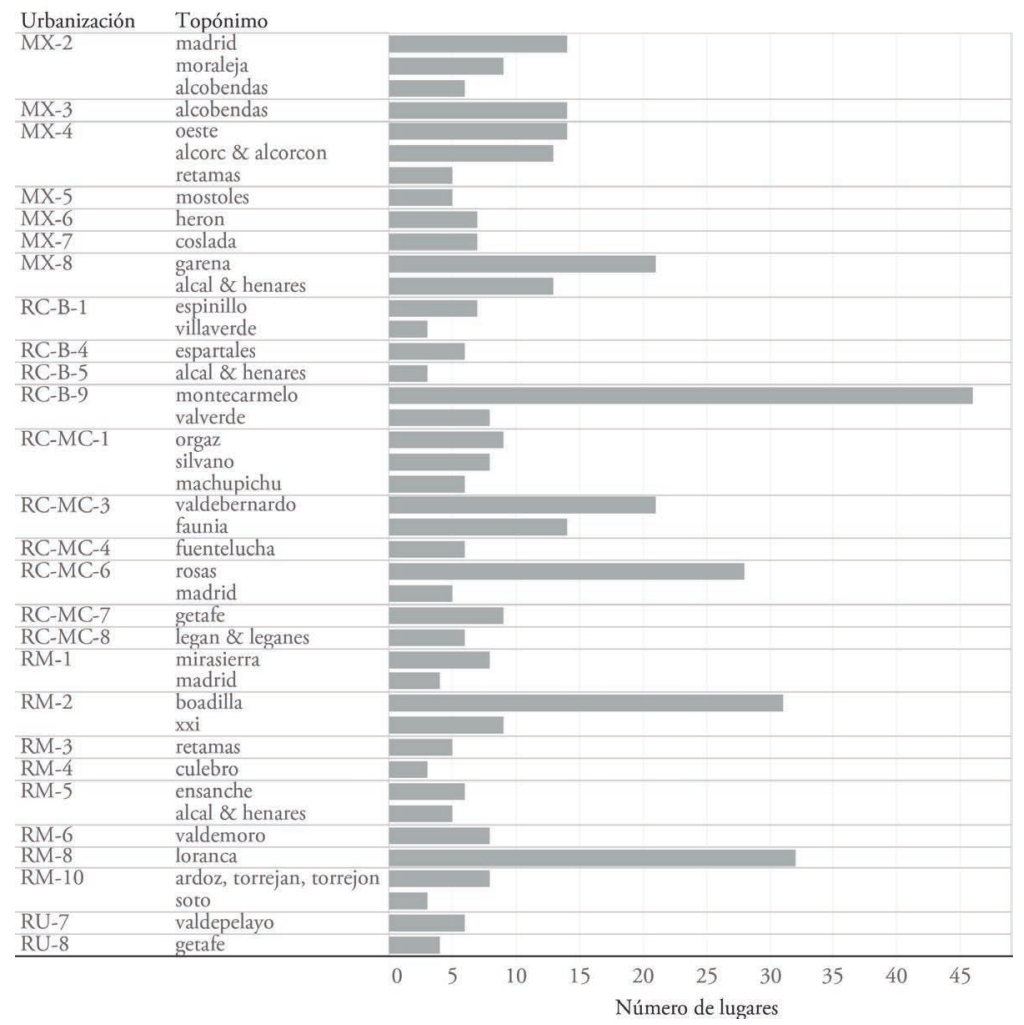

FIGURA 6 | Topónimos por frecuencia de repetición en el nombre de lugares específicos, y pieza en la que se encuentran

FUENTE: ELABORACIÓN PROPIA 


\section{Conclusiones}

Este trabajo planteaba un acercamiento a los procesos y los elementos productores de identidad y apropiación espacial en los entornos urbanos. Con foco espaciotemporal en el Madrid de la burbuja inmobiliaria española, tratamos de establecer una correspondencia entre la propia definición de apropiación espacial e identidad social urbana desde el ámbito de la psicología, y el análisis de datos cuantitativos y cualitativos de la red social Foursquare en cuanto a los lugares y sus características. De este modo, se trabaja con una metodología que triangula entre las teorías de la construcción de identidad, el estudio de actividad en la ciudad, y el análisis de datos espaciales.

Diversos aspectos relacionados con la generación de identidad y apropiación espacial se han podido extraer a partir del estudio de los datos, en correspondencia con aspectos morfológicos de las áreas estudiadas. La densidad edificada y la mixticidad de usos edificatorios desde el diseño tipológico se corresponden en términos generales con el volumen de datos asociados a cada pieza. Las actividades de tipo opcional de consumo, ocio o interacción social constituyen hitos en la mayoría de los casos, especialmente en el de grandes centros comerciales y de establecimientos de marcas reconocibles o franquicias. En algunos casos particulares, se corrobora una apropiación de los lugares ligada a la dimensión simbólica de los mismos, pero no necesariamente vinculada a la dimensión afectiva, sino a la asociación de valores e incorporación de los mismos en la definición intencionada de las narrativas individuales de los usuarios, como parte del spatial self. También se ha observado cómo la proximidad y la conexión de las piezas con tejidos preexistentes desempeña un papel fundamental; únicamente se construye una identidad nueva e independiente en piezas aisladas, y las piezas cercanas o diseñadas como expansión de otros tejidos se incorporan en las definiciones de estos. Asimismo, la red de transporte público tiene un papel fundamental en la consolidación de la identidad por medio de topónimos.

No obstante, se ha detectado una gran variedad de situaciones y resultados que, aunque permiten identificar ciertas tendencias, no siguen patrones únicos, confirmando que la cuestión de la identidad es sin duda compleja. El análisis de las nuevas fuentes de datos de gran volumen (más de 744.900 check-ins en este estudio) permite hacer una descripción amplia del conjunto y una comparación entre tipos de tejidos y piezas específicas a una escala que métodos tradicionales, como entrevistas y encuestas, no permiten. No obstante, la segmentación asociada al uso de redes sociales -y en particular al uso de Foursquare- limita sociodemográficamente la muestra con la que se trabaja. Al tratarse de un estudio con una componente social prioritaria, ha de tenerse en cuenta que para conocer los entresijos de la formación de identidad urbana, se requiere de métodos complementarios. Un estudio como el que se presenta aquí es sin duda útil para, i) establecer patrones generales y, ii) detectar casos singulares en los que se deben concentrar los esfuerzos de investigación con métodos más elaborados y pormenorizados (encuestas, entrevistas u observaciones in situ, entre otros), evitando el sesgo tecnológico asociado a las redes sociales o a los lugares con escaso volumen de datos asociados. Sin embargo, a pesar de estas limitaciones asociadas al método, se han observado patrones consistentes 
ligados a la correspondencia entre aspectos identitarios y de apropiación con la tipología morfológica, y sobre los cuales deberían apoyarse mejores propuestas urbanísticas en el futuro.

\section{Referencias bibliográficas}

Agryzkov, T., Nolasco-Cirugeda, A., Oliver, J. L., Serrano-Estrada, L., Tortosa, L., \& Vicent, J. F. (2015). Using data from Foursquare Web Service to represent the commercial activity of a city. International Journal of Computer, Control, Quantum and Information Engineering. World Academy of Science, Engineering and Technology, 9(1), 69-76. http:// waset.org/publications/10000159

Augé, M. (1994). Pour une anthropologie des mondes contemporains. Paris: Flammarion.

Barkhuus, L., Brown, B., Bell, M., Sherwood, S., Hall, M., \& Chalmers, M. (2008, April). From awareness to repartee: sharing location within social groups. En Proceedings of the SIGCHI conference on human factors in computing systems (497-506). ACM. https://doi. org/10.1145/1357054.1357134

Ben-Akiva, M. \& Bowman, J. L. (1998). Integration of an activity-based model system and a residential location model. Urban Studies, 35(7), 1131-1153. https://doi. org/10.1080/0042098984529

Bonnes, M. \& Secchiaroli, G. (1992). Psicologia ambientale: introduzione alla psicologia sociale e ambientale. Roma: Carocci.

Bourdieu, P. (1984). Distinction. A social critique of the judgement of taste. Oxon: Routledge.

Buttimer, A. \& Seamon, D. (1980). The human experience of sense and place. New York: Routledge. https://doi.org/10.4324/9781315684192

Cerrone, D., López Baeza, J., \& Lehtovuori, P. (2018). Integrative urbanism: using social media to map activity patterns for decision-making assessment. En H. van der Meer, G. Enthoven, \& G. Schiuma, (eds.), IFKAD 2018 Societal Impact of Knowledge and Design (pp. 1094-1107), 4-6 July 2018, Zelft, Netherlands, Delft University of Technology.

Duarte, F. (2002). Crise das matrizes espaciais - Arquitetura, cidades. geopolítica, tecnocultura. São Paulo: Perspectiva.

Duarte, F. (2017). Space, place and territory: A critical review on spatialities. New York: Routledge. https://doi.org/10.4324/9781315598888

Gehl, J. (1987). Life between buildings: using public space. Washington, DC; Covelo; London: Island Press.

Gibson, J. J. (1979). The ecological approach to visual perception. Boston: Houghton Mifflin Company. https://doi.org/10.2307/429816

Gretzel, U. \& Yoo, K. H. (2008). Use and impact of online travel reviews. Information and communication technologies in tourism 2008, 35-46. https://doi.org/10.1007/978-3211-77280-5_4

Jiang, S., Ferreira, J., \& González, M. C. (2012). Clustering daily patterns of human activities in the city. Data Mining and Knowledge Discovery, 25(3), 478-510. https://doi. org/10.1007/s10618-012-0264-z 
Jin, L., Zhang, K., Lu, J., \& Lin, Y. R. (2016). Towards understanding the gamification upon users' scores in a location-based social network. Multimedia Tools and Applications, 75(15), 8895-8919. https://doi.org/10.1007/s11042-014-2317-3

Kheiri, A., Karimipour, F., \& Forghani, M. (2015). Intra-urban movement flow estimation using location based social networking data, International Archives of the Photogrammetry, Remote Sensing \& Spatial Information Sciences, 40. https://doi.org/10.5194/ isprsarchives-XL-1-W5-781-2015

Komninos, A., Stefanis, V., Plessas, A., \& Besharat, J. (2013). Capturing urban dynamics with scarce check-in data. IEEE Pervasive Computing, 12(4), 20-28. https://doi.org/10.1109/ MPRV.2013.42

Lalli, M. (1992). Urban-related identity: theory, measurement, and empirical findings. Journal of Environmental Psychology, 12(4), 285-303. https://doi.org/10.1016/S02724944(05)80078-7

Lamíquiz Daudén, P. J., Pozueta Echavarri, J., Benito Moreno, M., \& González López, J. (2017). Desarrollos urbanos y transporte público ferroviario. El caso en la región metropolitana de Madrid: TODs, TADs y TJDs. Papers: Regió Metropolitana de Barcelona, (59), 58-76.

Lazzarini, L. \& López Baeza, J. (2016). The Mushrooms' Lesson: Instagram as a tool to evaluate users' perception of urban transformations. En Un nuovo ciclo della pianificazione urbanistica tra tattica e strategia (pp. 178-184). Roma/Milano: Planum Publisher.

Leal, J. \& Sorando, D. (2015). Economic crisis, social change and segregation processes in Madrid. En T. Tammaru, M. van Ham, S. Marcińczak, \& S. Musterd (eds.), SocioEconomic Segregation in European Capital Cities: East Meets West (pp. 214-237). Abingdon, uk: Routledge.

Lefebvre, H. (1974). La producción del espacio. Papers: Revista de Sociología, 3, 219-230. https://doi.org/10.5565/rev/papers/v3n0.880

López Baeza, J., Cerrone, D., \& Männigo, K. (2017). Comparing two methods for Urban Complexity calculation using Shannon-Wiener index. En wit Transactions on Ecology and Environment (pp. 369-378). Ashurst, Southhampton: Ashurst Lodge. https://doi. org/10.2495/SDP170321

López de Lucio, R. (2006). El tsunami urbanizador, la ciudad-basura y las arquitecturas de prestigio: Tres síntomas de la crisis urbana a comienzos del siglo xxI. Arquitectura: Revista del Colegio Oficial de Arquitectos de Madrid, 346, 86-89. http://oa.upm. es/45892/

López de Lucio, R. (2013). Vivienda colectiva, espacio público y ciudad. Buenos Aires: Nobuko. López de Lucio, R. \& Hernández Aja, A. (1995). Los nuevos ensanches de Madrid: la morfología residencial de la periferia reciente, 1985-1993. Madrid: Gerencia Municipal de Urbanismo.

Luke, R. (2005). The phoneur: mobile commerce and the digital pedagogies of the wireless web. En P. Trifonas (ed.), Communities of difference: culture, language, technology (pp. 185-204). London: Palgrave Macmillan.

Lynch, K. (1960). The image of the city. Chicago, Il: MIT Press.

Manovich, L. (2009). The practice of everyday (media) life: From mass consumption to mass cultural production? Critical Inquiry, 35(2), 319-331. https://doi.org/10.1086/596645 
Manovich, L. (2017). Aesthetics,'Formalism', and Media Studies. En L. Ouellette \& J. Gray (eds.), Keywords for Media Studies. New York: NYu Press.

Martí, P., Serrano-Estrada, L., \& Nolasco-Cirugeda, A. (2017). Using locative social media and urban cartographies to identify and locate successful urban plazas. Cities, 64, 66-78. https://doi.org/10.1016/j.cities.2017.02.007

Murillo, F. J. \& Martínez-Garrido, C. (2018). Magnitud de la segregación escolar por nivel socioeconómico en España y sus Comunidades Autónomas y comparación con los países de la Unión Europea. Revista de Sociología de la Educación (RASE), 11(1), 37-58. https://doi.org/10.7203/RASE.11.1.10129

Panerai, P., Castex, J., \& Depaule, J. C. (1997). Formes urbaines: de l'îlot à la barre. Marseille: Editions Parenthèses.

Pol, E. (1997). Symbolism a priori. Symbolism a posteriori. En A. Remesar (ed.), Urban regeneration. A challenge for public art (pp. 71-76). Barcelona: Publicacions de la Universitat de Barcelona.

Pozueta Echavarri, J., Benito, M., Carpio, J. González, J., \& Gómez, A. (en prensa). Lecciones urbanísticas de la burbuja inmobiliaria: dimensiones, costos y beneficios de las formas características del crecimiento urbano español. 1990-2006. Cuadernos de Investigación Urbanistica. Madrid: Escuela Técnica Superior de Arquitectura de Madrid.

Pozueta, J., Carpio J., Tumini I., \& González J. (en prensa). Evaluación ambiental, social, económica, de salud y de movilidad de las áreas residenciales características de la burbuja inmobiliaria madrileña, 1990-2012. Cuadernos de Investigación Urbanistica. Madrid: Escuela Técnica Superior de Arquitectura de Madrid.

Proshansky, H. M. (1976). Environmental psychology and the real world. American Psychologist, 31(4), 303. https://doi.org/10.1037/0003-066X.31.4.303

Proshansky, H. M. (1978). The city and self-identity. Environment and Behavior, 10(2), 147169. https://doi.org/10.1177/0013916578102002

Proshansky, H. M., Fabian, A. K., \& Kaminoff, R. (1983). Place-identity: Physical world socialization of the self. Journal of environmental psychology, 3(1), 57-83. https://doi. org/10.1016/S0272-4944(83)80021-8

Rapoport, A. (1977). Human aspects of urban form. Vol. 3. Oxford: Pergamon.

Roick, O. \& Heuser, S. (2013). Location based social networks - Definition, current state of the art and research agenda. Transactions in GIS, 17(5), 763-784. https://doi. org/10.1111/tgis. 12032

Saker, M. \& Evans, L. (2016). Everyday life and locative play: an exploration of Foursquare and playful engagements with space and place. Media, Culture \& Society, 38(8), 11691183. https://doi.org/10.1177/0163443716643149

Santos, M. (1978). Por uma geografia nova. São Paulo: Hucitec.

Schwartz, R. \& Halegoua, G. R. (2014). The spatial self: location-based identity performance on social media. New media \& society, 17(10), 1643-1660. https://doi. org/10.1177/1461444814531364

Seamon, D. (1979). A geography of the lifeworld: movement, rest \& encounter. London: Croom Helm. https://doi.org/10.4324/9781315715698 
Serrano-Estrada, L., Serrano Salazar, S., \& Álvarez Álvarez F. J. (2014). Las redes sociales y los SIG como herramientas para conocer las preferencias sociales en las ciudades turísticas: el caso de Benidorm. En Tecnologias de la información para nuevas formas de ver el territorio: XVI Congreso Nacional de Tecnologias de Información Geográfica (pp. 1005-1012), Alicante, 25 al 27 de junio de 2014. Alicante: Universidad de Alicante, Asociación Española de Geografía, Grupo de Tecnologías de la Información Geográfica.

Silva, T. H., Vaz De Melo P. O., Almeida J. M., \& Loureiro A. A. (2014). Large-scale study of city dynamics and urban social behavior using participatory sensing. Wireless Communications, IEEE, 21 (February), 42-51. https://doi.org/10.1109/MWC. 2014.6757896

Stokols, D. \& Shumaker S. (1981). People in places: A transactional view of settings. En J. H. Harvey, (ed.), Cognition, social behavior and the environment (pp. 441-488). Hillsdale, NJ: Erlbaum.

Tajfel, H. (1981). Human groups and social categories: Studies in Social Psychology. Cambridge: Cambridge University Press.

Valera, S. (1993). El simbolisme en la ciutat. Funcions de I'espai simbòlic urbà. Tesis Doctoral. Barcelona: Universitat de Barcelona, Departament de Psicologia Social.

Valera, S. \& Pol, E. (1994). El concepto de identidad social urbana: una aproximación entre la psicología social y la psicología ambiental. Anuario de Psicología, 62, 5-24. https:// bit.ly/2GkdYbr

Vidal i Moranta, T. \& Pol Urrutia, E. (2005). La apropiación del espacio: una propuesta teórica para comprender la vinculación entre las personas y los lugares. Anuario de Psicología, 36(3), 281-197. http://www.redalyc.org/articulo.oa?id=97017406003

Zhang, K. \& Pelechrinis, K. (2014). Understanding spatial homophily: the case of peer influence and social selection. En Proceedings of the 23rd international World Wide Web Conference. https://doi.org/10.1145/2566486.2567990

Zukin, S. (1996). Space and symbols in an age of decline. En Re-presenting the City (pp. 4359). London: Macmillan Education uk. https://doi.org/10.1007/978-1-349-24439-3 